\title{
A Comparison of Patella Retention versus Resurfacing for Moderate or Severe Patellar Articular Defects in Total Knee Arthroplasty: Minimum 5-year Follow-up Results
}

\author{
Seung Suk Seo, MD, Chang Wan Kim, MD and Sang Won Moon, MD \\ Department of Orthopaedics, Inje University Pusan Paik Hospital, Inje University College of Medicine, Busan, Korea
}

\begin{abstract}
Purpose: The purpose of this study is to assess the clinical and radiological results of patients who underwent patellar retention or resurfacing for moderate or severe patellar articular defects during total knee arthroplasty and evaluate the clinical efficacy of patellar resurfacing according to the articular defect of the patella.

Materials and Methods: From May 2003 to March 2006, 252 patients (277 cases) underwent total knee arthroplasty by one surgeon. Intraoperatively, we divided these patients into a moderate articular defect group (50-75\%: group I) and a severe articular defect group (75-100\%: group II) and randomly performed patellar resurfacing. The average age was 67.2 years. There were 234 female and 17 male patients. The average follow-up period was 74.6 months. Clinical outcomes were analyzed using the Knee Society (KS) knee score. Functional score, Hospital for Special Surgery (HSS) score, Feller patellar score and range of motion (ROM). Radiological outcomes were analyzed using the congruence angle, Insall-Salvati ratio and patella tilt angle.

Results: The KS knee score and functional score at the last follow-up were 84.4/73.1 in the retention group and 85.2/71.8 in the resurfacing group $(p=0.80, p=0.63)$ in group I. In group II, the values were $82.1 / 75.1$ and $87.0 / 71.2$, respectively $(p=0.51, p=0.26)$. The HSS score and Feller patella score were $86.7 / 20.3$ in the retention group and $84.3 / 21.7$ in the resurfacing group $(\mathrm{p}=0.31, \mathrm{p}=0.29)$ in group I. In group II, the values were 91.6/21.2 and $85.5 / 22.1$, respectively $(\mathrm{p}=0.37 / \mathrm{p}=0.30)$. The knee $\operatorname{ROM}(\mathrm{p}=0.36 / \mathrm{p}=0.41)$, congruence angle $(\mathrm{p}=0.22 / \mathrm{p}=0.16)$, Insall-Salvati ratio $(\mathrm{p}=0.16 / \mathrm{p}=0.21)$ and patella tilt angle ( $\mathrm{p}=0.12 / \mathrm{p}=0.19)$ were not statistically different between the two groups.

Conclusions: In this study, we could not find any correlations between the degree of patellar articular defect and patellar resurfacing in terms of the clinical and radiological results. Therefore, patellar articular defects is thought to be less meaningful in determining patellar resurfacing.
\end{abstract}

Key words: Knee, Total knee arthroplasty, Patellar articular defect, Patellar resurfacing.

\section{Introduction}

Total knee arthroplasty has been widely accepted as a useful

Received April 25, 2011; Revised June 7, 2011; Accepted June 14, 2011. Correspondence to: Chang Wan Kim, MD.

Department of Orthopaedics, Inje University Pusan Paik Hospital, Inje University College of Medicine,

633-165 Gaegeum 2-dong, Busanjin-gu, Busan 614-735, Korea.

Tel: +82-51-890-6257, Fax: +82-51-892-6619

Email: kcw8737@google.co.kr

This is an Open Access article distributed under the terms of the Creative Common Attribution Non-Commercial License (http://creativecommons.org/licenses/by-nc/3.0/) which permits unrestricted non-commercial use, distribution, and reproduction in any medium, provided the original work is properly cited.

Copyright $\odot$ 2011. THE KOREAN KNEE SOCIETY

www.jksrr.org treatment for degenerative osteoarthritis in terms of pain relief and return to normal activities, and considerable improvements have been made with regard to surgical techniques. However, the clinical efficacy of patellar resurfacing in total knee arthroplasty still remains controversial, and indications for patellar resurfacing have not been established. Patellar resurfacing appears more effective than patella retention with respect to pain relief, patient satisfaction, and complication rates in some studies ${ }^{1,2)}$ but it appears vice versa in other studies ${ }^{3-5)}$. In the meantime, some authors suggest that patellar resurfacing should be performed on a selective basis ${ }^{6-8)}$, which has recently been widely recognized.

Burnett and Bourne ${ }^{9)}$ reported that age, weight, gender, anatomical factors including the condition of the patellar cartilage, radiographic findings, and the presence of preoperative anterior knee pain and systemic diseases such as rheumatoid arthritis should be accounted for in determining whether 
patellar resurfacing is necessary. Among them, patellar cartilage condition has been considered as a major determinant of patellar resurfacing, and it has become a practice to resurface the patella based on the intraoperative assessment of a patellar cartilage defect ${ }^{10)}$. Although there have been some studies that suggest the opposite, most of those studies did not address clinical outcomes.

We conducted a retrospective study to investigate the correlation between the efficacy of patellar resurfacing in total knee arthroplasty and the patellar cartilage condition in patients with moderate or severe patellar cartilage defects. The patients were divided into two groups, the patellar resurfacing group and the retention group, and clinical and radiological outcomes of patellar resurfacing were assessed.

\section{Materials and Methods}

Of the patients who had undergone total knee arthroplasty for osteoarthritis by the same surgeon at our institution between May 2003 and March 2006, 252 patients (277 cases) with intraoperative findings of moderate or severe patellar cartilage defects that involve $\geq 50 \%$ of the patellar articular surface (Outerbridge grade III-IV) were enrolled in the study (Fig. 1). The patients were divided into two groups, the retention group and the resurfacing group, for comparison. In 25 patients with a bilateral total knee arthroplasty, the patella was resurfaced in one knee and retained in the other. To calculate the cartilage loss in percentage, the size of a defect measured directly during surgery and the image captured with a digital camera positioned perpendicular to the articular surface were processed by computer program. There were 109 knees in the retention group and 168 knees in the resurfacing group. The knees with 50 -
$75 \%$ cartilage loss were classified into group 1 and those with $\geq 75 \%$ cartilage loss into group II. Patients were randomized to treatment with or without patellar resurfacing. In group 1, the patella was retained in 96 knees and resurfaced in 145 knees. In group 2, the patella was retained in 13 knees and resurfaced in 23 knees. The mean age at the time of surgery was 67.2 years (range, 42 to 82 years). There were 235 females and 17 males. The mean follow-up period was 74.6 months (range, 60 to 93 months).

The exclusion criteria included a valgus or varus angle of $\geq 15^{\circ}$ on the low extremity orthoscanogram, a congruence angle of $>16^{\circ}$, a patella tilt angle of $>5^{\circ}$, an abnormal Insall-Salvati ratio, a lateral retinacular release during total knee arthroplasy, patellar dislocation, and systemic arthritis including rheumatoid arthritis.

The arthroplasty was performed via a medial parapatellar approach using the E-motion posterior cruciate ligament retaining (B.Braun, Tuttilingen, Germany) prosthesis in all patients. The femoral and tibial components were cemented in all knees. The horizontal and vertical lengths of the patellar cartilage and of the defect were measured (Fig. 1). Based on the measurements, the patients were divided into those with $50-75 \%$ cartilage loss and those with $\geq 75 \%$ loss. In the resurfacing group, a symmetrical dome-shaped cemented all-polyethylene patellar component was used. In the retention group, osteophytes around the patella were removed but cartilage debridement, abrasion arthroplasty, and multiple perforations were not performed.

Clinical evaluation was performed using the Knee Society knee (KS knee) score, functional score, Hospital for Special Surgery (HSS) score, Feller patellar score, and range of joint motion preoperatively and postoperatively. The range of joint motion was measured by an independent investigator who was unaware of the study using a goniometer: the active range of joint motion
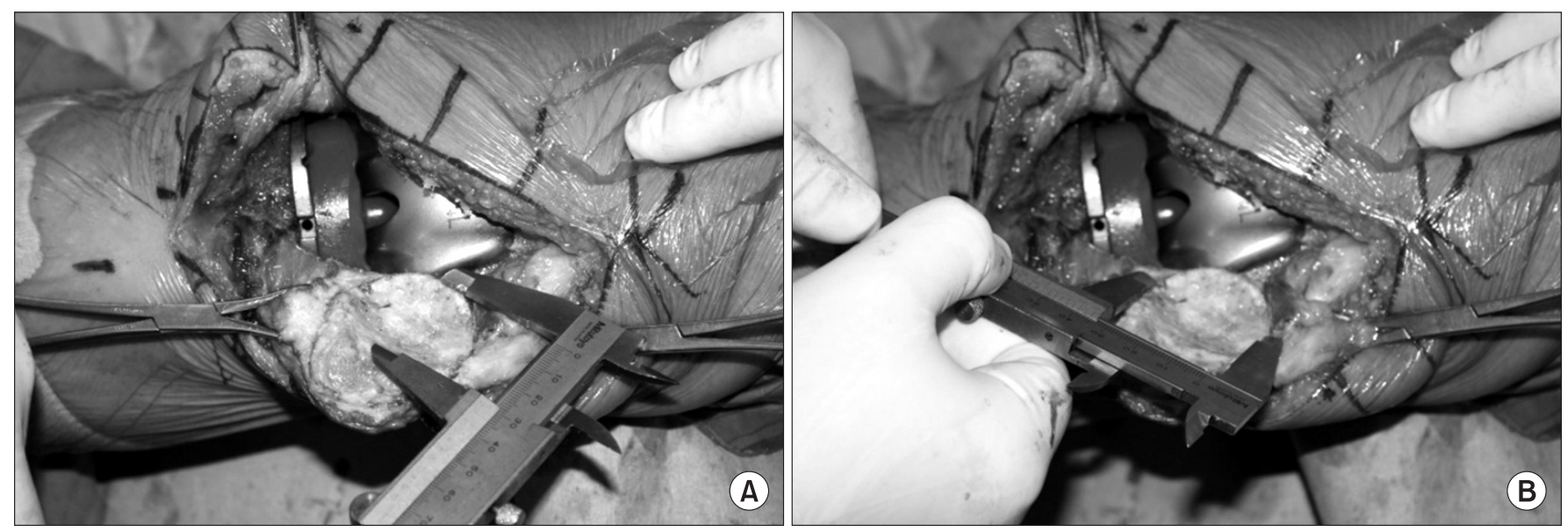

Fig. 1. These photos show how to measure patellar articular defect in total knee arthroplasty. (A) This photo shows how to measure the horizontal length of the cartilage defect of the patella. (B) This photo shows how to measure the vertical length of the cartilage defect of the patella. 

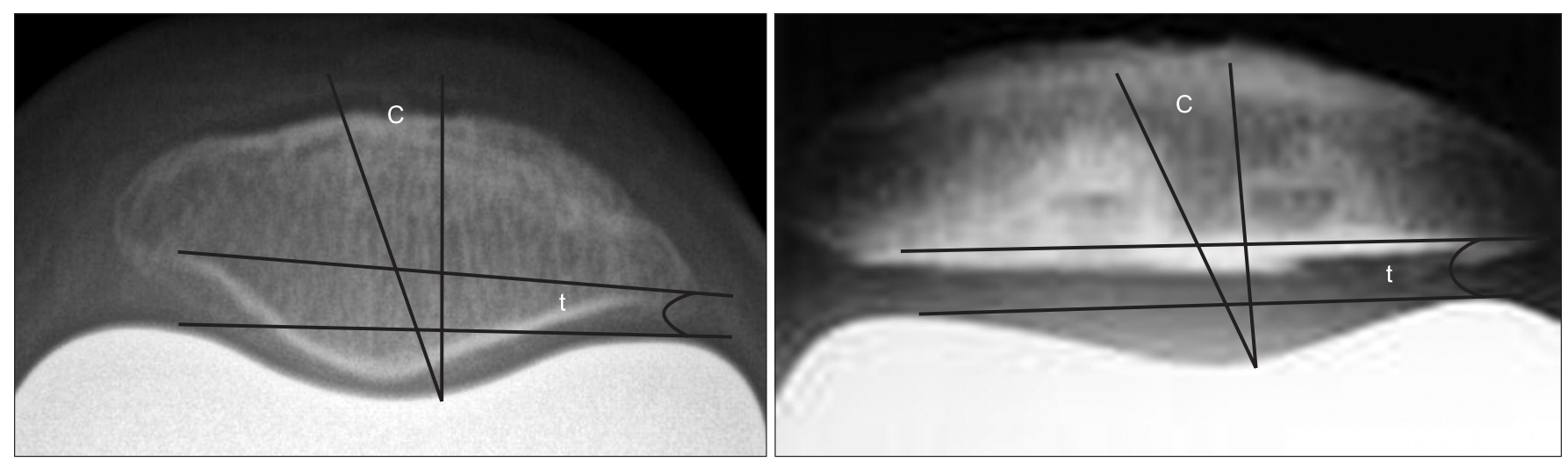

Fig. 2. Congruence angle (c) and Patella tilt angle (t) are not statistically different in the two groups. If the congruence angle is lateral to the congruence line, it is a positive congruence angle and if is medial, it is a negative congruence angle. If the coronal axis of the patella is laterally tilted to the tangent line of the medial and lateral trochlear ridge, it is a positive patella tilt angle and if it is medially tilted, it is a negative patella tilt angle.
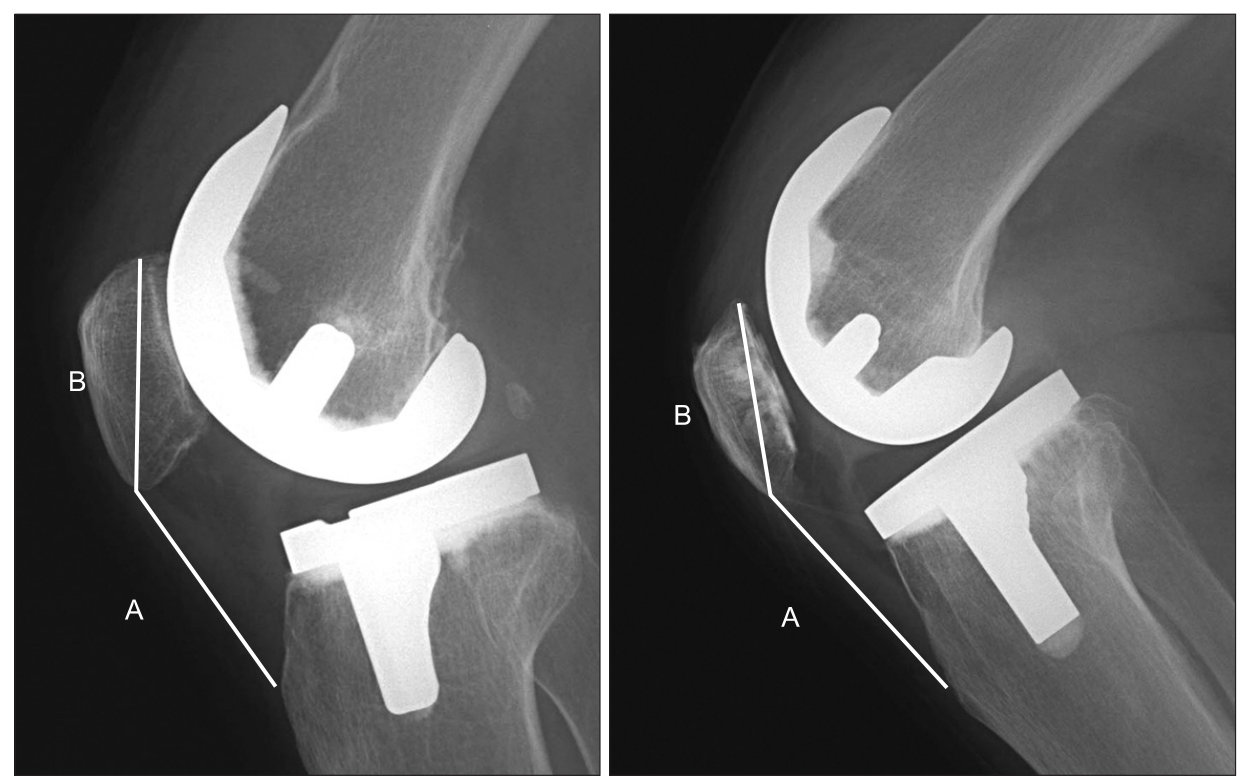

Fig. 3. Insall-Salvati ratio $(A / B)$ is not statistically different in the two groups.

was measured in one-degree increments with one arm of the goniometer aligned parallel to the long axis from the greater trochanter to the lateral epicondyle and the other arm from the fibular head to the lateral malleolus of the fibula. Radiological assessment was performed using the congruence angle, patella tilt angle (Fig. 2), and Insall-Salvati ratio (Fig. 3). Two independent radiologists were asked to analyze the data three times each using the PACS (Marosis-Infinitt Co., Seoul, Korea) data to reduce the intra- and inter-observer variability. Statistical analysis was performed at a 95\% confidence level using a Wilcoxon rank-sum test (ver. 11.5, SPSS Inc., Chicago, IL, USA).

\section{Results}

Preoperatively, there was no statistically significant difference between the groups with respect to age, height, weight, BMI, range of knee joint motion, KS Knee score, functional score, HSS score, Feller patellar score, congruence angle, Insall-Salvati ratio, patella tilt angle, and anterior knee pain (Table 1).

\section{Clinical Outcomes}

1) KS knee score and functional score

The mean KS Knee score at the last follow-up was 83.2 in the retention group with 84.4 in group I (50-75\% involvement) and 82.1 in group II ( $\geq 75 \%$ involvement). The score was 86.1 ( $\mathrm{p}=0.79)$ in the resurfacing group with $85.2(\mathrm{p}=0.80)$ in group I and 87.0 
Table 1. Demographics

\begin{tabular}{lccc}
\hline & Retention & Resurfacing & p-value \\
\hline Age $(\mathrm{y})$ & $67.1(42-82)$ & $67.2(42-82)$ & 0.21 \\
Height $(\mathrm{cm})$ & $152.5(142-168)$ & $153.3(142-168)$ & 0.34 \\
Weight $(\mathrm{kg})$ & $61.0(37.6-85.2)$ & $61.5(37.6-85.2)$ & 0.12 \\
Body mass index $\left(\mathrm{kg} / \mathrm{m}^{2}\right)$ & $26.8(18.9-37.0)$ & $26.4(18.9-37.0)$ & 0.17 \\
Congruence angle $\left(^{\circ}\right)$ & $-5.0(-16.8-9.2)$ & $-4.9(-16.8-9.2)$ & 0.28 \\
Insall-Salvati ratio & $1.1(0.77-1.34)$ & $1.1(0.77-1.34)$ & 0.42 \\
Patella tilt angle $\left(^{\circ}\right)$ & $4.1(0-12)$ & $1.8(0-12)$ & 0.18 \\
Knee Society Knee score & $32.1(0-48)$ & $35.2(0-48)$ & 0.71 \\
Functional score & $49.3(30-60)$ & $45.6(30-60)$ & 0.13 \\
Hospital for Special Surgery score & $36.6(0-50)$ & $37.1(0-50)$ & 0.57 \\
Feller patellar score & $16.8(10-30)$ & $16.7(10-30)$ & 0.31 \\
Anterior knee pain & 46 & 72 & 0.10 \\
\hline
\end{tabular}

Table 2. Knee Society Knee Score/Functional Score

\begin{tabular}{lccc}
\hline & Group I & Group II & I + II \\
\hline Retention & $84.4 / 73.1$ & $82.1 / 75.1$ & $83.2 / 74.1$ \\
Resurfacing & $85.2 / 71.8$ & $87.0 / 71.2$ & $86.1 / 71.5$ \\
\hline
\end{tabular}

Table 3. Hospital for Special Surgery Score/Feller Patellar Score

\begin{tabular}{lccc}
\hline & Group I & Group II & I + II \\
\hline Retention & $86.7 / 20.3$ & $91.6 / 21.2$ & $89.1 / 20.7$ \\
Resurfacing & $84.3 / 21.7$ & $85.5 / 22.1$ & $84.9 / 21.9$ \\
p-value & $0.31 / 0.29$ & $0.37 / 0.30$ & $0.43 / 0.36$ \\
\hline
\end{tabular}

$(\mathrm{p}=0.51)$ in group II. The mean functional score at the last followup was 74.1 in the retention group with 73.1 in group I and 75.1 in group II. The score was $71.5(\mathrm{p}=0.51)$ in the resurfacing group with $71.8(\mathrm{p}=0.68)$ in group I and $71.2(\mathrm{p}=0.26)$ in group II. The KS knee score and functional score were significantly improved compared to the preoperative values. However, no statistically significant differences were found between the retention group and resurfacing group and between groups I and II classified according to the cartilage defect (Table 2).

\section{2) HSS score and feller patellar score}

The mean HSS score at the last follow-up was 89.1 in the retention group with 86.7 in group I and 91.6 in group II. The score was $84.9(\mathrm{p}=0.43)$ in the resurfacing group with $84.3(\mathrm{p}=0.31)$ in group I and $85.5(\mathrm{p}=0.37)$ in group II. The mean Feller patellar score at the last follow-up was 20.7 in the retention group with 20.3 in group I and 21.2 in group II. The
Table 4. Knee Range of Joint Motion

\begin{tabular}{lccc}
\hline & Group I & Group II & I+II \\
\hline Retention $\left(^{\circ}\right)$ & 131.2 & 130.8 & 131 \\
Resurfacing $\left({ }^{\circ}\right)$ & 132.3 & 131.4 & 131.8 \\
p-value & 0.42 & 0.33 & 0.64 \\
\hline
\end{tabular}

score was $21.9(\mathrm{p}=0.36)$ in the resurfacing group with 21.7 $(\mathrm{p}=0.29)$ in group I and $22.1(\mathrm{p}=0.30)$ in group II. The HSS score and Feller patellar score were significantly improved compared to the preoperative values. However, no statistically significant differences were found between the retention group and resurfacing group and between groups I and II classified according to the cartilage defect (Table 3).

\section{3) Range of joint motion (ROM)}

There was no statistically significant difference between the retention group and resurfacing group before surgery. The mean knee ROM at the last follow-up was $131^{\circ}$ with $131.2^{\circ}$ in group I and $130.8^{\circ}$ in group II. The value in the resurfacing group was $131.8^{\circ}(\mathrm{p}=0.64)$ with $132.3^{\circ}(\mathrm{p}=0.42)$ in group I and $131.4^{\circ}$ $(\mathrm{p}=0.33)$ in group II. No statistically significant differences were found between the retention group and resurfacing group and between groups I and II classified according to the cartilage defect (Table 4).

\section{4) Complications}

Anterior knee pain occurred in 2 knees of the retention group and in 1 knee of the resurfacing group. Loosening of the knee prosthesis was not observed. 
Table 5. Congruence Angle/Insall-Salvati Ratio/Patella Tilt Angle

\begin{tabular}{lccc}
\hline & Group I & Group II & I + II \\
\hline Retention & $-12.8^{\circ} / 1.13 / 4.8^{\circ}$ & $-9.8^{\circ} / 1.29 / 4.6^{\circ}$ & $-11.3^{\circ} / 1.21 / 4.7^{\circ}$ \\
Resurfacing & $-2.8^{\circ} / 1.12 / 1.7^{\circ}$ & $-5.6^{\circ} / 1.09 / 1.6^{\circ}$ & $-4.2^{\circ} / 1.10 / 1.7^{\circ}$ \\
p-value & $0.22 / 0.58 / 0.58$ & $0.16 / 0.85 / 0.47$ & $0.23 / 0.76 / 0.45$ \\
\hline
\end{tabular}

\section{Radiological Outcomes}

The mean congruence angle at the last follow-up was $-11.3^{\circ}$ in the retention group with $-12.8^{\circ}$ in group I and $-9.8^{\circ}$ in group II. The value was $-4.2^{\circ}(\mathrm{p}=0.23)$ in the resurfacing group with $-2.8^{\circ}$ $(\mathrm{p}=0.22)$ in group I and $-5.6^{\circ}(\mathrm{p}=0.16)$ in group II. The mean Insall-Salvati ratio at the last follow-up was 1.21 in the retention group with 1.13 in group I and 1.29 in group II. The value was $1.10(\mathrm{p}=0.76)$ in the resurfacing group with $1.12(\mathrm{p}=0.58)$ in group I and $1.09(\mathrm{p}=0.85)$ in group II. The mean patella tilt angle at the last follow-up was $4.7^{\circ}$ in the retention group with $4.8^{\circ}$ in group I and $4.6^{\circ}$ in group II. The value was $1.7^{\circ}(\mathrm{p}=0.45)$ in the resurfacing group with $1.7^{\circ}(\mathrm{p}=0.58)$ in group I and $1.6^{\circ}(\mathrm{p}=0.47)$ in group II. No statistically significant differences were found between the retention group and resurfacing group and between groups I and II classified according to the cartilage defect (Table 5).

\section{Discussion}

There is still controversy regarding patella resurfacing in total knee arthroplasty. Patella resurfacing had not been performed in the early years of total knee arthroplasty and it was introduced as a solution to anterior knee pain later ${ }^{11)}$. However, it has been associated with early complications including patellar fracture and component loosening that have been attributed to poor patellar component designs and surgical techniques ${ }^{12)}$. Although some recent studies suggest improvements in that regard, there are still other studies reporting no improvements in terms of postoperative complications and treatment outcomes ${ }^{9)}$. The current consensus is that patellar resurfacing should be performed on a selective basis. In the past, the presence of preoperative anterior knee pain was thought as one of the major indications for patellar resurfacing. However, it has been elucidated that posterior anterior knee pain does not necessarily appear secondary to knee resurfacing and other criteria for knee surfacing play a key role in the occurrence of the pain ${ }^{9}$. Currently, the degree of patellar cartilage defect rather than the presence of preoperative anterior knee pain is of primary importance in determining patellar resurfacing ${ }^{13)}$.
Indeed, Picetti et al. ${ }^{14)}$ suggested that patellar resurfacing could result in more satisfactory results in knees with moderate or severe patellar cartilage defects based on their study of 100 cases of total knee arthroplasty. Enis ${ }^{15)}$ reported that patellar resurfacing was more effective in relieving pain and restoring strength after bilateral total knee arthroplasty in 25 patients with moderate or severe patellar cartilage defects in whom the patella was resurfaced in one knee and retained in the other.

In contrast, Burnett et al. ${ }^{4}$ reported that their survey showed no difference between the resurfacing group and the retention group in terms of patient satisfaction, the presence of anterior knee pain, and knee joint function. Braakman et al. ${ }^{16)}$ could not find differences between the resurfacing group and retention group with regard to the range of movement, pain, stability, walking distance, ability to climb stairs, patellofemoral complications, and the frequency of revision surgery. According to Barrack et al. ${ }^{17)}$, the occurrence of anterior knee pain following knee joint arthroplasty was more associated with the location of prosthesis and surgical technique than patellar resurfacing and it could not be predicted with radiographic parameters. Kulkarni et al. ${ }^{18)}$ followed 267 patients who had undergone total knee arthropalsty for 10-15 years and concluded that major prognostic factors of patellar resurfacing are the design of the prosthetic trochlea and surgical technique rather than the condition of the patellar cartilage.

Similarly, we could not find a significant correlation between the degree of patellar cartilage defect and clinical rand radiological outcomes of patellar resurfacing in patients with moderate or severe patellar cartilage defects. Kyung et al. ${ }^{13)}$ suggested that clinical improvements cannot be expected when patellar resurfacing is determined based on the intraoperative assessment of the patellar cartilage defect and degenerative condition in total knee arthroplasty. This is because although early postoperative anterior knee pain and snapping occurred proportional to the degree of patellar cartilage defect, the differences disappeared and so did the symptoms over time. In addition, they could not find a correlation between the Feller patellar score and the degree of patellar cartilage defect. Han et al. ${ }^{19)}$ examined 80 cases of total knee arthroplasty without patellar resurfacing and suggested that the depth and size of of the patellar articular cartilage lesion were not correlated with the symptoms and function of the patella and the location of the lesion was not correlated with the patellofemoral function. Cho et al. ${ }^{20)}$ performed total knee arthroplasty without patellar resurfacing regardless of the preoperative radiographic findings of degeneration and could obtain satisfactory clinical and radiological outcomes. Song et 
$\mathrm{al}^{21)}$ reported satisfying results of total knee arthroplasty without patellar resurfacing regardless of the patellar articular cartilage condition at a minimum of 5-year follow-up. These studies support our finding that patellofemoral cartilage defect was an appropriate indication for patellar resurfacing in total knee arthroplasty.

The significance of our study is that we evaluated the impact of moderate or severe patellofemoral articular cartilage defect on the clinical and radiological outcomes of patellar resurfacing in total knee arthroplasty by comparing the patellar retention group and resurfacing group for the 74-month follow-up period.

\section{Conclusions}

We could not find significant clinical and radiological differences between patellar resurfacing and patellar retention in total knee arthroplasty in patients with moderate or severe patellofemoral articular cartilage defects. Patellar cartilage defect that had been considered as an important determinant for patellar resurfacing had no influence on clinical and radiological outcomes. Therefore, it is our understanding that patellar articular cartilage defect could not be an important factor in determining whether patellar resurfacing is necessary.

\section{References}

1. Cameron HU, Jung YB. A comparison of unicompartmental knee replacement with total knee replacement. Orthop Rev. 1988;17:983-8.

2. Heck DA, Marmor L, Gibson A, Rougraff BT. Unicompartmental knee arthroplasty. A multicenter investigation with long-term follow-up evaluation. Clin Orthop Relat Res. 1993;(286):154-9.

3. Barrett WP, Scott RD. Revision of failed unicondylar unicompartmental knee arthroplasty. J Bone Joint Surg Am. 1987;69:1328-35.

4. Burnett RS, Haydon CM, Rorabeck CH, Bourne RB. Patella resurfacing versus nonresurfacing in total knee arthroplasty: results of a randomized controlled clinical trial at a minimum of 10 years' followup. Clin Orthop Relat Res. 2004;(428):12-25.

5. Hernigou P, Deschamps G. Patellar impingement following unicompartmental arthroplasty. J Bone Joint Surg Am. 2002;84:1132-7.

6. Dalury DF, Ewald FC, Christie MJ, Scott RD. Total knee arthroplasty in a group of patients less than 45 years of age. J
Arthroplasty. 1995;10:598-602.

7. Garneti N, Mahadeva D, Khalil A, McLaren CA. Patellar resurfacing versus no resurfacing in Scorpio total knee arthroplasty. J Knee Surg. 2008;21:97-100.

8. Waters TS, Bentley G. Patellar resurfacing in total knee arthroplasty. A prospective, randomized study. J Bone Joint Surg Am. 2003;85:212-7.

9. Burnett RS, Bourne RB. Indications for patellar resurfacing in total knee arthroplasty. Instr Course Lect. 2004;53:167-86.

10. Kim BS, Reitman RD, Schai PA, Scott RD. Selective patellar nonresurfacing in total knee arthroplasty. 10 year results. Clin Orthop Relat Res. 1999;(367):81-8.

11. Gunston FH, MacKenzie RI. Complications of polycentric knee arthroplasty. Clin Orthop Relat Res. 1976;(120):11-7.

12. Bayley JC, Scott RD, Ewald FC, Holmes GB Jr. Failure of the metal-backed patellar component after total knee replacement. J Bone Joint Surg Am. 1988;70:668-74.

13. Kyung HS, Kim SJ, Lee SM, Ihn JC. Functional and radiological outcomes of patella retaining total knee arthroplasty: minimun 5-year follow-up results. J Korean Orthop Assoc. 2002;37:337-41.

14. Picetti GD 3rd, McGann WA, Welch RB. The patellofemoral joint after total knee arthroplasty without patellar resurfacing. J Bone Joint Surg Am. 1990;72:1379-82.

15. Enis JE. Comparison of patellar resurfacing versus nonresurfacing in bilateral total knee arthroplasty. Clin Orthop Relat Res. 1993;(288):310.

16. Braakman $M$, Verburg AD, Bronsema $G$, van Leeuwen WM, Eeftinck MP. The outcome of three methods of patellar resurfacing in total knee arthroplasty. Int Orthop. 1995;19:711.

17. Barrack RL, Bertot AJ, Wolfe MW, Waldman DA, Milicic M, Myers L. Patellar resurfacing in total knee arthroplasty. A prospective, randomized, double-blind study with five to seven years of follow-up. J Bone Joint Surg Am. 2001;83:1376-81.

18. Kulkarni SK, Freeman MA, Poal-Manresa JC, Asencio JI, Rodriguez JJ. The patello-femoral joint in total knee arthroplasty: is the design of the trochlea the critical factor? Knee Surg Sports Traumatol Arthrosc. 2001;9(Suppl 1):S812.

19. Han I, Chang CB, Lee S, Lee MC, Seong SC, Kim TK. Correlation of the condition of the patellar articular cartilage and patellofemoral symptoms and function in osteoarthritic patients undergoing total knee arthroplasty. J Bone Joint Surg Br. 2005;87:1081-4. 
20. Cho SH, Chung UH, Chun CW, Yune YP. Total Knee Arthroplasty without patellar resurfacing. J Korean Knee Soc. 2002;14:111-6.
21. Song EK, Yoon TR, Jung JW, Kim JS. Clinical and radiological results of total knee arthroplasty with patellar retention. J Korean Knee Soc. 2001;13:137-41. 